\title{
Exacerbation action plans for patients with COPD and comorbidities: a randomised controlled trial
}

\author{
Anke Lenferink ${ }^{1,2,3}$, Job van der Palen ${ }^{1,4}$, Paul D.L.P.M. van der Valk ${ }^{1}$, \\ Paul Cafarella ${ }^{3,5}$, Anneke van Veen ${ }^{6}$, Stephen Quinn ${ }^{7}$, \\ Catharina G.M. Groothuis-Oudshoorn ${ }^{2}$, Morton G. Burt ${ }^{3,8}$, Mary Young ${ }^{9}$, \\ Peter A. Frith $\mathbb{1}^{3}$ and Tanja W. Effing ${ }^{3,5}$
}

@ERSpublications

Patient-tailored exacerbation action plans for COPD patients with comorbidities do not reduce exacerbation days, but reduce exacerbation duration and risk of having at least one respiratory-related hospitalisation during follow-up, without excess mortality http://bit.ly/2Mi8Fhc

Cite this article as: Lenferink A, van der Palen J, van der Valk PDLPM, et al. Exacerbation action plans for patients with COPD and comorbidities: a randomised controlled trial. Eur Respir J 2019; 54: 1802134 [https://doi.org/10.1183/13993003.02134-2018].

ABSTRACT This international randomised controlled trial evaluated whether COPD patients with comorbidities, trained in using patient-tailored multidisease exacerbation action plans, had fewer COPD exacerbation days than usual care (UC).

COPD patients (Global Initiative for Chronic Obstructive Lung Disease (GOLD) classification II-IV) with $\geqslant 1$ comorbidity (ischaemic heart disease, heart failure, diabetes, anxiety, depression) were randomised to a patient-tailored self-management intervention $(n=102)$ or UC ( $n=99)$. Daily symptom diaries were completed for 12 months. The primary outcome "COPD exacerbation days per patient per year" was assessed using intention-to-treat analyses.

No significant difference was observed in the number of COPD exacerbation days per patient per year (self-management: median 9.6 (interquartile range (IQR) 0.7-31.1); UC: median 15.6 (IQR 3.0-40.3); incidence rate ratio (IRR) 0.87 (95\% CI $0.54 ; 1.39) ; \mathrm{p}=0.546)$. There was a significantly shorter duration per COPD exacerbation for self-management (self-management: median 8.1 (IQR 4.8-10.1) days; UC: median 9.5 (IQR 7.0-15.1) days; $\mathrm{p}=0.021$ ), with no between-group differences in the total number of respiratory hospitalisations (IRR $0.76(95 \%$ CI $0.42 ; 1.35) ; \mathrm{p}=0.348)$, but a lower probability of $\geqslant 1$ for respiratory-related hospitalisation compared to UC (relative risk 0.55 (95\% CI 0.35; 0.87); $\mathrm{p}=0.008$ ). No between-group differences were observed in all-cause hospitalisations (IRR 1.07 (95\% CI 0.66; 1.72)) or mortality (self-management: $\mathrm{n}=4$ (3.9\%); UC: $\mathrm{n}=7$ (7.1\%); relative risk 0.55 (95\% CI 0.17; 1.84)).

Patient-tailored exacerbation action plans for COPD patients with comorbidities did not significantly reduce exacerbation days, but reduced the duration per COPD exacerbation and the risk of having at least one respiratory-related hospitalisation during follow-up, without excess all-cause mortality.

This study is registered in the public Australian New Zealand Clinical Trials Registry (ACTRN12612000514808). All relevant data are presented within the paper and its supplementary files. All data are from the COPE-III study, whose authors may be contacted at Medisch Spectrum Twente, Dept of Pulmonary Disease, Enschede, The Netherlands. A copy of the patient-tailored diary and action plan can be requested from the corresponding author. Furthermore, detailed methods including a statistical analysis plan are published elsewhere.

This article has supplementary material available from erj.ersjournals.com

Received: 06 April 2018 | Accepted after revision: 22 July 2019

Copyright OERS 2019 


\section{Introduction}

COPD is a progressive lung condition with distressing exacerbations that contribute to impaired quality of life and increased hospitalisations, mortality and healthcare costs. Many COPD patients have comorbidities [1,2] that further reduce quality of life [3] and are associated with an increased risk of COPD exacerbations, hospitalisations, mortality and higher costs $[1,2,4,5]$. Evidence for COPD self-management interventions and action plans that are tailored for comorbidities is lacking.

A COPD self-management intervention is defined as "structured, but personalised, and often multicomponent, with goals of motivating, engaging and supporting the patients to positively adapt their health behaviour(s) and develop skills to better manage their disease" [6]. COPD exacerbation action plans are an integral part of COPD self-management interventions and have proven to reduce exacerbation duration, hospitalisations and healthcare costs, and to improve quality of life in COPD patients without comorbidities [7-10]. Benefits arise from earlier initiation of appropriate treatment, decreasing the severity of exacerbations, accelerating recovery and reducing healthcare utilisation [11, 12]. However, it is challenging to generalise these results to routine clinical practice in the wider COPD population, as most self-management studies have excluded COPD patients with comorbidities. The applicability, effectiveness and safety of symptom-based COPD action plans may be limited when comorbidities are present, as comorbid symptoms may overlap with COPD symptoms, leading to the initiation of incorrect or delayed treatment. Hence, the need for evidence-based exacerbation action plans that are tailored for existing comorbidities [13].

In the COPE-III study [14] we designed patient-tailored action plans (supplementary material) for both COPD and comorbidities (ischaemic heart disease (IHD), chronic heart failure (CHF), diabetes mellitus (DM), anxiety, depression) [14, 15]. A copy can be requested from the corresponding author. We evaluated whether patients with COPD and $\geqslant 1$ comorbidity, trained in using patient-tailored symptom-based exacerbation action plans embedded in a multidisease self-management intervention, had fewer COPD exacerbation days over 12 months than usual care (UC) [14].

\section{Methods}

Detailed methods, including a statistical analysis plan of this 1-year international multicentre open parallel-group randomised controlled trial (RCT), are published elsewhere [14]. The study was approved by the Medical Ethical Committee Twente (NL39516.044.12) and the Southern Adelaide Clinical Human Research Ethics Committee (37-12). Participants' written informed consent was obtained prior to data collection.

\section{Patients}

Between 2012 and 2015, patients were recruited from the outpatient departments of respiratory medicine of two hospitals in the Netherlands (Medisch Spectrum Twente, Enschede; Canisius-Wilhelmina Ziekenhuis, Nijmegen) and three in Australia (Repatriation General Hospital, Adelaide; Royal Adelaide Hospital, Adelaide; Flinders Medical Centre, Adelaide). Patients with COPD and one to five highly prevalent comorbidities were selected for this study. These comorbidities were chosen because of symptom overlap with COPD exacerbations, and/or their potential to become unstable during COPD exacerbations: IHD (history of myocardial infarction, angina pectoris); CHF (2008 ESC guidelines [16]), DM (glucocorticoid-induced, or stable type 1 or 2 ); active symptoms of anxiety and/or depression $(\geqslant 11$ Hospital Anxiety and Depression Scale (HADS) [17, 18]), and/or anxiety or depression symptoms being treated at the time of inclusion.

After baseline measurements, patients were allocated to self-management or UC by an independent research assistant, who was blinded to the treatment assignment and randomisation schedule, using a computerised minimisation program [19]. Allocation was stratified per hospital for smoking status,

Affiliations: ${ }^{1}$ Dept of Pulmonary Medicine, Medisch Spectrum Twente, Enschede, The Netherlands. ${ }^{2}$ Dept of Health Technology and Services Research, Faculty of Behavioural, Management and Social Sciences, Technical Medical Centre, University of Twente, Enschede, The Netherlands. ${ }^{3}$ College of Medicine and Public Health, Flinders University, Adelaide, Australia. ${ }^{4}$ Dept of Research Methodology, Measurement, and DataAnalysis, Faculty of Behavioural, Management and Social Sciences, University of Twente, Enschede, The Netherlands. ${ }^{5}$ Dept of Respiratory Medicine, Southern Adelaide Local Health Network, Adelaide, Australia. ${ }^{6}$ Dept of Pulmonary Medicine, Canisius-Wilhelmina Ziekenhuis, Nijmegen, The Netherlands. ${ }^{7}$ Dept of Statistics, Data Science and Epidemiology, Swinburne University of Technology, Melbourne, Australia. ${ }^{8}$ Southern Adelaide Diabetes and Endocrine Services, Flinders Medical Centre, Adelaide, Australia. ${ }^{9}$ Dept of Thoracic Medicine, Royal Adelaide Hospital, Adelaide, Australia.

Correspondence: Anke Lenferink, Dept of Pulmonary Medicine, Medisch Spectrum Twente, P.0. Box 50 000, 7500 KA Enschede, The Netherlands. E-mail: a.lenferinkamst.nl 
modified Medical Research Council dyspnoea (mMRC) score, number of comorbidities and being on a waiting list for pulmonary rehabilitation. Blinding of patients and personnel to the treatment group was not possible. Wherever possible, though, assessors of outcomes were blinded to the treatment group.

At inclusion all patients described their individual symptom levels in a stable health state on a "What are my 'usual' symptoms" card and were educated to complete daily symptom diaries for 12 months (supplementary material). The colour-coded patient-tailored diaries included symptoms of COPD and comorbidities diagnosed at baseline [14]. On each day of the study patients were asked to compare their symptoms in the last $24 \mathrm{~h}$ to their "usual" symptoms, and, if symptom levels had increased, to report the level of change for each symptom listed in their diary (i.e. no change, slightly increased, or significantly increased). Completed diaries including information regarding unscheduled healthcare visits and use of additional medications were returned at the end of each month. Incomplete diary data were compared with hospital admission data. Subsequently, patients were phoned to complete missing diary data, to provide feedback on diary use, to adjust symptom levels on the "What are my 'usual' symptoms" card if necessary (e.g. after an exacerbation), and to reinforce self-management behaviours (self-management group only). For the self-management group, exacerbation action plans for COPD, CHF, IHD, anxiety and depression were directly linked to the diaries. For diabetes, this link was indirect, as patients were prompted to check their blood glucose levels when the COPD action plan directed them to take glucocorticoid because of a COPD exacerbation. Additional details on the self-management and UC sessions are provided in table 1 (supplementary material).

\section{Outcomes}

The total number of COPD exacerbation days per patient per year was defined as the primary outcome. We also analysed the number of COPD exacerbations per patient per year, the duration per COPD exacerbation per patient per year and the severity of a COPD exacerbation day. COPD exacerbation data were collected from the symptom diaries. The onset was defined as a "clear negative change in two symptoms classified major (dyspnoea, sputum purulence, sputum volume) or one major and one minor symptom (coughing, wheezing, fever) from baseline, for $\geqslant 2$ consecutive days" $[20,21]$. Recovery was

\section{TABLE 1 Content and components of self-management and usual care sessions}

Self-management intervention group

Week 1 Group session:

- knowledge regarding COPD and comorbidities

- symptom recognition and monitoring

- self-treatment (action plan linked to diary)

- breathing and relaxation exercises

- Dutch patients with diabetes: extra session on how to check and regulate blood glucose levels when necessary

- Australian patients with diabetes: extra session on how to check blood glucose levels when necessary

Week 2 Individual session:

- complete "What are my 'usual' symptoms" card

- diary training

- exacerbation action plan training

- mastery of skills; e.g. correct inhaler techniques (re-iterated when necessary), early recognition of exacerbations, self-initiating correct and proper actions

Week 3 Group session:

- importance of physical fitness and exercise

- diet and lifestyle behaviours

- re-iteration of diary and action plan use

- re-iteration of breathing and relaxation exercises

Week 4 Individual session:

- re-iteration of diary and action plan use

- feedback on diary completion

- feedback on actions

Week 8 Phone call $1^{\text {II }}$

Week 20 Phone call 2"

Week 36 Phone call $3^{\text {Il }}$

\section{Usual care control group}

Group session:

- complete "What are my "usual" symptoms" card

- diary training

Group session:

- re-iteration of use of diary

- feedback on diary completion

Phone call $1^{\#}$

Phone call $2^{\#}$

Phone call $3^{\#}$

Phone call $4^{\#}$

Phone call $5^{\#}$

\#: approximately 5-10 min each; ": approximately 10-15 min each. 
defined as "the first day of: 1) three consecutive successive days that the patient has returned to his or her normal baseline health state; or 2) seven consecutive days on which patients continuously reported no or a slightly increase of COPD symptoms compared to baseline". The duration per COPD exacerbation was calculated by dividing the number of COPD exacerbation days by the number of COPD exacerbations. The severity of a COPD exacerbation day was determined by the sum of the individual relative changes from baseline daily symptom scores for major and minor COPD symptoms (for algorithm refer to supplementary material). Other secondary outcomes are described in detail elsewhere [14] and summarised in table 2.

\section{Statistical analysis}

Based on the exacerbation rates/patient/day from the COPE-II study [9] (intervention: 0.116; control: 0.176 , both with an estimated standard deviation of 0.17 ) and allowing for overdispersion and an attrition rate of $10 \%, 105$ patients per group provided $80 \%$ power to detect an effect of this size, with a two-tailed $\mathrm{p}<0.05$.

Analyses were conducted on an intention-to-treat basis and reported in accordance with CONSORT guidelines [22]. Differences in baseline characteristics between self-management and UC were analysed using common statistical procedures, such as chi-square tests, t-tests and Mann-Whitney U-tests, as appropriate.

If there were fewer than four consecutive days of missing diary data, these were completed using a pre-defined algorithm that combined the last observation carried forward and the next observation carried backward to the missing value. Since these data were considered to be missing completely at random, this single imputation technique was considered valid [23]. If four or more consecutive diary days were missing, the missing entries were not considered to be completely at random. Therefore, a negative binomial regression imputation method was used to complete these diary days for the overdispersed individual patient count variable (COPD exacerbation days). This parametric method assumes an underlying negative binomial model for the imputed variable (given other predictors).

\begin{tabular}{|c|c|c|c|}
\hline & $\begin{array}{l}\text { Baseline } \\
\text { (hospital) }\end{array}$ & $\begin{array}{l}6 \text { months (per } \\
\text { mail) }\end{array}$ & $\begin{array}{l}12 \text { months } \\
\text { (hospital) }\end{array}$ \\
\hline Baseline characteristics & $x$ & & \\
\hline $\begin{array}{l}\text { Post-bronchodilator spirometry }\left(\mathrm{FEV}_{1}, \mathrm{FEV}_{6} \text {, }\right. \\
\text { FVC) }\end{array}$ & $x$ & & $x$ \\
\hline COPD Assessment Test & $x$ & & $x$ \\
\hline mMRC dyspnoea score & $X$ & $X$ & $x$ \\
\hline \multicolumn{4}{|l|}{ Health-related quality of life } \\
\hline EQ-5D and VAS & $X$ & $X$ & $x$ \\
\hline $\mathrm{CRQ}$ & $x$ & $x$ & $x$ \\
\hline \multicolumn{4}{|l|}{ Subjective fatigue } \\
\hline ICFS & $x$ & $x$ & $x$ \\
\hline \multicolumn{4}{|l|}{ Anxiety and depression symptoms } \\
\hline HADS & $x$ & $x$ & $x$ \\
\hline \multicolumn{4}{|l|}{ Confidence and competence } \\
\hline CSES & $x$ & $x$ & $X$ \\
\hline $\mathrm{CRQ}$ mastery domain & $x$ & $x$ & $x$ \\
\hline \multicolumn{4}{|l|}{$\begin{array}{l}\text { Patient self-management behaviour and } \\
\text { knowledge }\end{array}$} \\
\hline $\mathrm{PIH}$ scale & $X$ & & $\mathrm{X}$ \\
\hline \multicolumn{4}{|l|}{ Cost and healthcare utilisation } \\
\hline $\begin{array}{l}\text { Healthcare resource use for } 12 \text { months, EQ-5D } \\
\text { for utilities }\end{array}$ & & & $x$ \\
\hline \multicolumn{4}{|l|}{$\begin{array}{l}\text { Patient adherence to action plans, satisfaction } \\
\text { and confidence }\end{array}$} \\
\hline $\begin{array}{l}\text { Semi-structured interviews and daily symptom } \\
\text { diary (retrospective) }\end{array}$ & & & $x$ \\
\hline
\end{tabular}

CRQ: Chronic Respiratory Disease Questionnaire; CSES: COPD Self-Efficacy Scale; EQ-5D: EuroQol 5 dimensions; $\mathrm{FEV}_{1}$ : forced expiratory volume in $1 \mathrm{~s} ; \mathrm{FEV}_{6}$ : forced expiratory volume in $6 \mathrm{~s}$; FVC: forced (expiratoryl vital capacity; HADS: Hospital Anxiety Depression Scale; ICFS: Identity-Consequence Fatigue Scale; mMRC: modified Medical Research Council; PIH: Partners in Health; VAS: Visual Analogue Scale. 
TABLE 3 Baseline characteristics of the self-management intervention and usual care group

\begin{tabular}{|c|c|c|}
\hline & $\begin{array}{l}\text { Self-management } \\
\qquad(n=102)\end{array}$ & $\begin{array}{l}\text { Usual care } \\
\qquad(n=99]\end{array}$ \\
\hline Age years & $68.8 \pm 9.0$ & $68.2 \pm 8.9$ \\
\hline Males & $66(64.7 \%)$ & $63(63.6 \%)$ \\
\hline Smoker & $20(19.6 \%)$ & $20(20.2 \%)$ \\
\hline COPD exacerbations 2 years prior to study participation & $3.0(2.0-4.0)$ & $3.0(2.0-4.0)$ \\
\hline Hospitalisations 1 year prior to study participation & $1.0(0-1.0)$ & $1.0(0-1.0)$ \\
\hline Smoking history pack-years & $41.0(22.0-52.8)$ & $37.3(24.8-53.8)$ \\
\hline Body mass index $\mathrm{kg} \cdot \mathrm{m}^{-2}$ & $29.8 \pm 6.8$ & $29.6 \pm 6.4$ \\
\hline Dyspnoea score (mMRC) & $2.17 \pm 1.02$ & $2.04 \pm 1.10$ \\
\hline \multicolumn{3}{|l|}{ Post-bronchodilator spirometry } \\
\hline $\mathrm{FEV}_{1} \mathrm{~L}$ & $1.42 \pm 0.53$ & $1.36 \pm 0.50$ \\
\hline $\mathrm{FEV}_{1} \%$ pred & $53.4 \pm 16.1$ & $50.7 \pm 14.3$ \\
\hline $\mathrm{FEV}_{1} / \mathrm{FVC} \%$ & $49.3 \pm 14.3$ & $48.5 \pm 12.2$ \\
\hline \multicolumn{3}{|l|}{ GOLD COPD classification } \\
\hline GOLD II & $60(58.8 \%)$ & $54(54.5 \%)$ \\
\hline GOLD III & 35 (34.3\%) & $39(39.4 \%)$ \\
\hline GOLD IV & $7(6.9 \%)$ & $6(6.1 \%)$ \\
\hline BODE score $^{+}$ & $3.31 \pm 2.11$ & $3.32 \pm 2.18$ \\
\hline 6-min walking distance ${ }^{\S}$ & $330 \pm 116$ & $378 \pm 112^{\#}$ \\
\hline Self-reported exercise $\mathbf{h}$ per week ${ }^{f}$ & $4.0(2.0-8.0)$ & $5.0(2.0-10.0)$ \\
\hline \multicolumn{3}{|l|}{ Education level } \\
\hline Not completed primary school & $4(3.9 \%)$ & $5(5.1 \%)$ \\
\hline Primary education & $14(13.7 \%)$ & $11(11.1 \%)$ \\
\hline Not completed secondary school & $38(37.3 \%)$ & $26(26.3 \%)$ \\
\hline Secondary education & $18(17.6 \%)$ & $23(23.2 \%)$ \\
\hline Trade school & $22(21.6 \%)$ & $25(25.3 \%)$ \\
\hline Undergraduate & $1(1 \%)$ & $3(3 \%)$ \\
\hline Postgraduate & $4(3.9 \%)$ & $4(4 \%)$ \\
\hline Unknown & $1(1 \%)$ & $2(2 \%)$ \\
\hline Cognitive impairment (MMSE) & $28.5(27.0-29.0)$ & $29.0(26.0-30.0)$ \\
\hline Health literacy confidence & $49(48.0 \%)$ & $57(57.6 \%)$ \\
\hline COPD-specific health status (CAT) ๆๆ & $19.4 \pm 5.6$ & $18.0 \pm 7.2$ \\
\hline General health status (VAS) & $60.9 \pm 14.3$ & $60.6 \pm 17.9$ \\
\hline \multicolumn{3}{|l|}{ Quality of life domains (CRQ) } \\
\hline Dyspnoea & $4.25 \pm 1.38$ & $4.22 \pm 1.49$ \\
\hline Fatigue & $3.77 \pm 1.15$ & $3.84 \pm 1.28$ \\
\hline Emotional & $4.71 \pm 1.26$ & $4.65 \pm 1.07$ \\
\hline Mastery & $4.89 \pm 1.16$ & $4.90 \pm 1.14$ \\
\hline Self-management knowledge and behaviour $(\mathrm{PIH})^{++}$ & $77.9 \pm 10.3$ & $78.4 \pm 10.3$ \\
\hline Knowledge and coping & $35.1 \pm 7.3$ & $35.5 \pm 7.2$ \\
\hline Symptom management, adherence to treatment & $42.9 \pm 4.5$ & $42.9 \pm 4.4$ \\
\hline \multicolumn{3}{|l|}{ Self-efficacy domains (CSES) } \\
\hline Negative affects & $1.72 \pm 0.90$ & $1.54 \pm 0.77$ \\
\hline Emotional arousal & $1.61 \pm 0.83$ & $1.50 \pm 0.82$ \\
\hline Physical exertion & $2.47 \pm 0.82$ & $2.36 \pm 0.89$ \\
\hline Environment & $2.02 \pm 0.78$ & $1.95 \pm 0.87$ \\
\hline Behavioural risk factors & $1.76 \pm 0.97$ & $1.58 \pm 0.80$ \\
\hline Fatigue (ICFS) & $224.4 \pm 81.7$ & $220.8 \pm 80.8$ \\
\hline Experiences & $47.8 \pm 16.8$ & $45.9 \pm 15.5$ \\
\hline Impacts & $40.5 \pm 18.1$ & $41.6 \pm 18.1$ \\
\hline \multicolumn{3}{|l|}{ Anxiety and depression domains (HADS) } \\
\hline Anxiety symptoms & $6.94 \pm 4.39$ & $6.86 \pm 3.82$ \\
\hline Depression symptoms & $6.78 \pm 3.95$ & $6.60 \pm 3.87$ \\
\hline Specified comorbidities & $1.61 \pm 0.79$ & $1.58 \pm 0.77$ \\
\hline Adapted Charlson index $§ \S$ & $2.61 \pm 0.83$ & $2.70 \pm 0.86$ \\
\hline Ischaemic heart disease & $43(42.2 \%)$ & $50(50.5 \%)$ \\
\hline Chronic heart failure & $24(23.5 \%)$ & $23(23.2 \%)$ \\
\hline Anxiety ff & $27(26.5 \%)$ & $19(19.2 \%)$ \\
\hline
\end{tabular}


TABLE 3 Continued

\begin{tabular}{lcc} 
& $\begin{array}{c}\text { Self-management } \\
\text { (n=102) }\end{array}$ & $\begin{array}{c}\text { Usual care } \\
\text { (n=99) }\end{array}$ \\
\hline Depression $^{f f}$ & $31(30.4 \%)$ & $26(26.3 \%)$ \\
Diabetes mellitus & $39(38.2 \%)$ & $39(39.4 \%)$ \\
\hline
\end{tabular}

Data are presented as $\mathrm{n}(\%)$, mean $\pm \mathrm{SD}$ or median (interquartile range). BODE: Body mass index, obstruction, dyspnoea, exercise capacity; CAT: COPD Assessment Test; CRQ: Chronic Respiratory Disease Questionnaire; CSES: COPD Self-Efficacy Scale; FEV 1 : forced expiratory volume in $1 \mathrm{~s}$; FVC: forced (expiratory) vital capacity; GOLD: Global Initiative for Chronic Obstructive Lung Disease; HADS: Hospital Anxiety Depression Scale; ICFS: Identity-Consequence Fatigue Score; mMRC: modified Medical Research Council dyspnoea scale; MMSE: Mini Mental State Examination; PIH: Partners in Health scale; VAS: Visual Analogue Scale. Health literacy was measured by asking patients for their confidence in completing medical forms by themselves. " : significantly different $(p<0.01)$. ๆ: smoking history: self-management $n=99$; usual care $n=96 .{ }^{+}$: BODE score: self-management $n=99$; usual care $n=97 .{ }^{\S}$ : 6-min walking distance: self-management $n=94$; usual care $n=85 .{ }^{f}$ : self-reported exercise: self-management $n=94$; usual care $n=89$. \#\#: health literacy confidence: self-management $n=100$; usual care $n=97$. 17 : COPD-specific health status (CAT): self-management $n=99$; usual care $n=95 .{ }^{++}$: self-management knowledge and behaviour $(P I H)$ : self-management $n=101$; usual care $n=98$. $\S$ : we used an adapted version of the Charlson Index to classify comorbidities. Only the comorbidities included in our study were classified and scored as follows: pulmonary disease $=1$; angina $=1$; chronic heart failure $=1$; myocardial infarction $=1$; diabetes mellitus $=1$; anxiety $=1$; depression $=1$. Self-management $n=89$; usual care $n=88$. ${ }^{f f}$ : active symptoms of anxiety and/or depression (cut-off score $\geqslant 11$ from the HADS), and/or anxiety or depression symptoms being treated at the time of inclusion.

Analyses of the total number of COPD exacerbation days per patient per year (primary outcome) and COPD exacerbations per patient per year were conducted in three steps (supplementary material), using imputed data in negative binomial regression models. The imputed values for missing diary days were calculated based on: 1) the observed number of COPD exacerbation days or exacerbations; 2) variables that correlated to exacerbation days or exacerbations; and 3) variables that explained missing days (supplementary material). Incidence rate ratios (IRRs) were calculated. Sensitivity analyses were conducted by including only observed data (without any imputations). The between-group difference in duration per COPD exacerbation was analysed using a Mann-Whitney U-test. The results for between-group differences at 12 months of follow-up for comorbid exacerbations (CHF, anxiety, depression, IHD events) are presented in the supplementary material. Between-group differences of secondary continuous variables over the 12-month period were assessed using linear mixed-effect models for repeated measurement analyses. Relative risks were calculated for the probability of $\geqslant 1$ hospitalisation and analysed using Z-tests.

All analyses were carried out using SPSS version 24.0 with $\mathrm{p}<0.05$ considered to be statistically significant.

\section{Results \\ Patients}

In total, 201 patients with moderate to severe COPD were included (mean \pm SD age $68.5 \pm 8.9$ years; $64.2 \%$ male; $19.9 \%$ current smokers; $1.6 \pm 0.8$ specified comorbidities at baseline) (table 3). At baseline, there were no significant differences between self-management $(n=102)$ and UC $(n=99)$ groups, except for the 6-min walking distance (6MWD) (self-management: $330 \pm 116 \mathrm{~m}$; UC: $378 \pm 112 \mathrm{~m} ; \mathrm{p}=0.006$ ). The 12 -month follow-up was completed by $83.3 \%$ of the individuals in the self-management group and $84.8 \%$ in the UC group (figure 1). Patients completed $81.3 \%$ (self-management: $82.3 \%$; UC: $80.2 \%$ ) of the diary data.

\section{COPD exacerbations}

A total of 446 COPD exacerbations (self-management $n=216$; UC $n=230$ ) were extracted from the diary data. $66(64.7 \%)$ self-management patients had at least one COPD exacerbation compared to $70(70.7 \%)$ UC patients (relative risk 0.92 (95\% CI 0.76; 1.11); p=0.363) (table 4). There was no statistically significant difference in the median number of COPD exacerbation days per patient per year (self-management: median 9.6 (interquartile range (IQR) 0.7-31.1) days; UC: median 15.6 (IQR 3.0-40.3) days; IRR 0.87 (95\% CI $0.54 ; 1.39) ; \mathrm{p}=0.546)$. Sensitivity analyses including only observed diary data rendered similar results (table 4). Excluding patients with an extremely long COPD exacerbation duration ( $>300$ days) and thus a potential chronic deterioration of symptoms showed comparable results. 


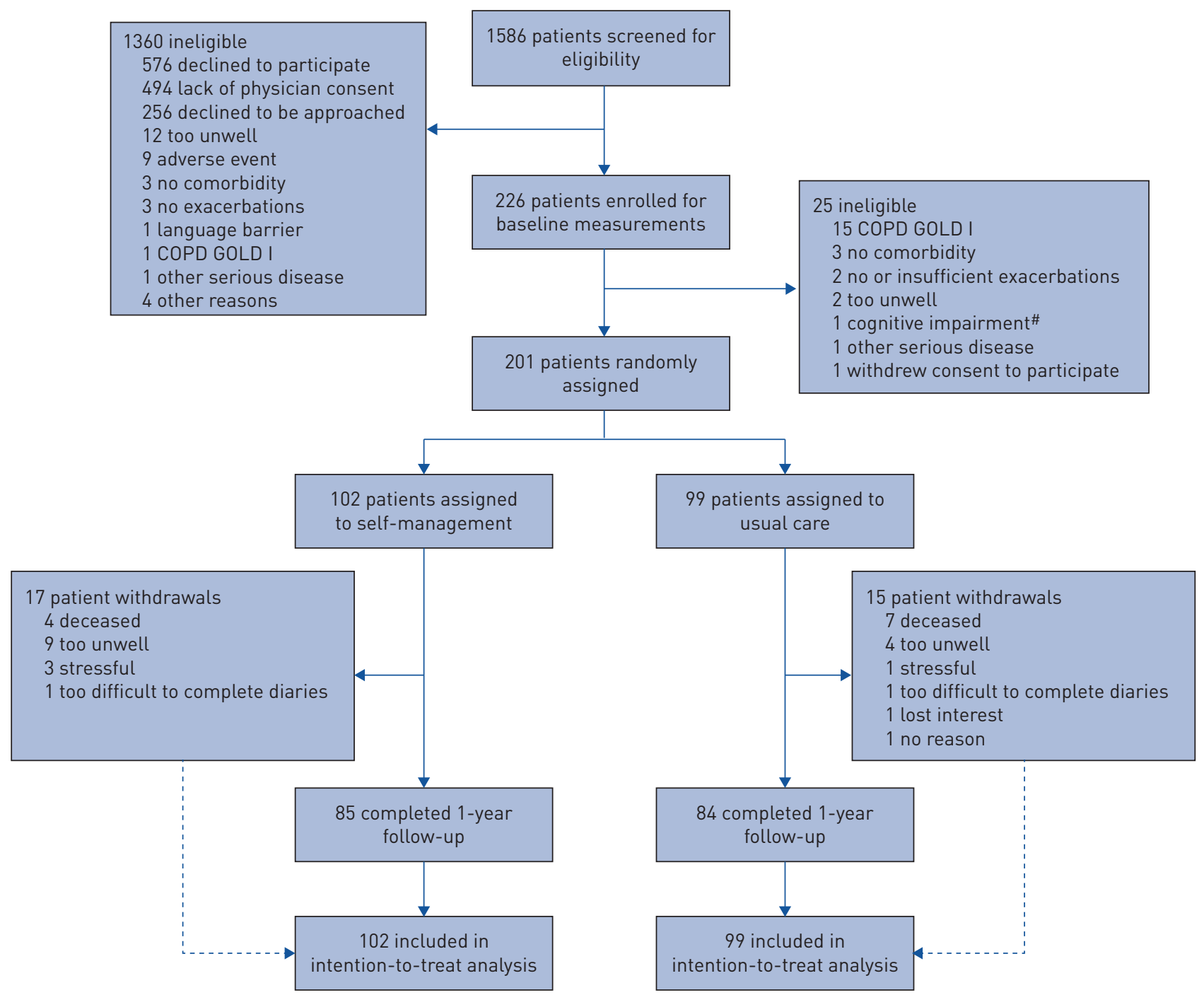

FIGURE 1 Flowchart of patient enrolment in the COPE-III study. "\#: Mini Mental State Examination <24.

A statistically significant and clinically relevant shorter duration per COPD exacerbation was found for self-management (median 8.1 (IQR 4.8-10.1) days) compared to UC patients (median 9.5 (IQR 7.0-15.1) days) ( $\mathrm{p}=0.021)$. Sensitivity analyses including only observed diary data showed similar results (table 4 ).

There was no difference in severity per COPD exacerbation day (self-management: median 4.1 (IQR 06.0); UC: median 5.0 (IQR 0-9.0); $\mathrm{p}=0.068$ ) (table 4).

\section{Hospitalisations}

There were 106 (self-management $\mathrm{n}=47$; UC $\mathrm{n}=59$ ) respiratory-related hospitalisations (mean $\pm \mathrm{SD} 0.53 \pm$ 1.09) reported (table 5). The self-management group had a lower probability of $\geqslant 1$ respiratory-related hospitalisation compared to UC (relative risk 0.55 (95\% CI $0.35 ; 0.87$ ); p=0.008), with no between-group differences in duration per respiratory-related hospitalisation (self-management: $8.68 \pm 6.5$ days; UC: $7.34 \pm$ 4.4 days; $\mathrm{p}=0.570$ ) and in total respiratory hospital admission rates (IRR 0.76 (95\% CI 0.42; 1.35); $\mathrm{p}=0.348$ ). The total number of respiratory-related hospitalisation days per patient was numerically lower in the self-management group, but this difference was not statistically significant (table 5).

At baseline 125 patients were diagnosed with CHF and/or IHD, in whom 20 cardiac-related hospitalisations were observed. No between-group differences were observed in cardiac-related hospitalisations (self-management: $n=12$; UC: $n=8 ; p=0.215$ ).

A total of 151 all-cause hospitalisations were extracted from diary data and medical records. No between-group differences in the number of all-cause hospitalisations were observed, nor in the total 
TABLE 4 COPD exacerbations: results from negative binomial regression and Mann-Whitney $U$ analyses for between-group differences at 12 months of follow-up

\begin{tabular}{|c|c|c|c|}
\hline \multirow{2}{*}{$\begin{array}{l}\text { Self-management } \\
\qquad(n=102)\end{array}$} & \multirow{2}{*}{$\begin{array}{l}\text { Usual care } \\
\qquad(n=99)\end{array}$} & \multicolumn{2}{|c|}{ Treatment effect (self-management versus usual care) } \\
\hline & & $\begin{array}{l}\text { IRR }(95 \% \mathrm{CI}) \text { or relative risk }(95 \% \mathrm{CI}) \text { or } \\
\text { difference in duration }\end{array}$ & \\
\hline
\end{tabular}

\begin{tabular}{|c|c|c|c|c|}
\hline \multicolumn{5}{|l|}{ COPD exacerbations } \\
\hline Median (IQR) & $1.1(0.1-3.9)$ & $2.0(0.7-4.0)$ & & \\
\hline Rate (per 100 person-years) & 2.4 & 2.7 & $0.91(0.67 ; 1.25)$ & $0.558^{\#}$ \\
\hline \multicolumn{5}{|l|}{ COPD exacerbation days } \\
\hline Median (IQR) & $9.6(0.7-31.1)$ & $\begin{array}{l}15.6(3.0- \\
40.3)\end{array}$ & & \\
\hline Rate (per 100 person-years) & 28.7 & 34.2 & $0.87(0.54 ; 1.39)$ & $0.546^{\#}$ \\
\hline Patients with $\geqslant 1$ COPD exacerbation & $n=66$ & $n=71$ & $0.90(0.75 ; 1.09)$ & $0.286^{+}$ \\
\hline $\begin{array}{l}\text { Duration per COPD exacerbation } \\
\text { (days), median (IQR) }\end{array}$ & $8.1(4.8-10.1)$ & $9.5(7.0-15.1)$ & -1.4 & $0.021^{\Uparrow}$ \\
\hline \multicolumn{5}{|l|}{ COPD exacerbations (observed) } \\
\hline \multicolumn{5}{|l|}{ COPD exacerbations } \\
\hline Median (IQR) & $1(0-3)$ & $2(0-3)$ & & \\
\hline Rate (per 100 person-years) & 2.1 & 2.3 & $0.91(0.65 ; 1.26)$ & $0.561^{\#}$ \\
\hline \multicolumn{5}{|l|}{ COPD exacerbation days } \\
\hline Median (IQR) & $9(0-27.5)$ & $13(0-39)$ & & \\
\hline Rate (per 100 person-years) & 24.9 & 28.7 & $0.90(0.56 ; 1.43)$ & $0.640^{\#}$ \\
\hline Patients with $\geqslant 1$ COPD exacerbation & $n=66$ & $n=70$ & $0.92(0.76 ; 1.11)$ & $0.363^{+}$ \\
\hline $\begin{array}{l}\text { Duration per COPD exacerbation } \\
\text { (days), median (IQR) }\end{array}$ & $7.7(4.3-10.0)$ & $9.4(6.5-14.6)$ & -1.7 & $0.012^{\Uparrow}$ \\
\hline $\begin{array}{l}\text { Severity per COPD exacerbation day, } \\
\text { median (IQR) }\end{array}$ & $4.1(0-6.0)$ & $5.0(0-9.0)$ & & $0.068^{\text {9 }}$ \\
\hline
\end{tabular}

Data are presented as median (interquartile range (IQR)) per patient per year or rate (per 100 person-years). IRR: incidence rate ratio.

\#: between-group difference results at 12 months of follow-up were obtained with negative binomial regression analyses; " non-parametric Mann-Whitney U-test for data related to duration; ${ }^{+}$: Z-test.

TABLE 5 Hospitalisations during 12 months of follow-up in the self-management and usual care group

\begin{tabular}{cccc}
\begin{tabular}{c} 
Self-management $\begin{array}{c}\text { Un=102) } \\
\text { (n=99) }\end{array}$ \\
\cline { 3 - 4 }
\end{tabular} & $\begin{array}{c}\text { Treatment effect (self-management versus usual } \\
\text { care })\end{array}$ \\
\cline { 3 - 4 } & $\begin{array}{c}\text { IRR }(95 \% \mathrm{CI}) \text { or relative risk }(95 \% \mathrm{CI}) \text { or } \\
\text { difference in duration }\end{array}$ & p-value
\end{tabular}

\begin{tabular}{|c|c|c|c|c|}
\hline \multicolumn{5}{|c|}{ Respiratory-related hospitalisations (primary respiratory-related problem) } \\
\hline Number of hospitalisations ( $\mathrm{n}$; mean $\pm s \mathrm{~d}$ ) & $47 ; 0.46 \pm 1.15$ & $59 ; 0.60 \pm 1.03$ & & \\
\hline Rate (per 100 person-years) & 46.1 & 59.6 & $0.76(0.42 ; 1.35)$ & $0.348^{\#}$ \\
\hline Days rate (per 100 person-years) & 3.8 & 4.3 & $0.92(0.49 ; 1.73)$ & $0.789^{\#}$ \\
\hline $\begin{array}{l}\text { Duration per hospitalisation days } \\
\text { (mean } \pm \text { SD)] }\end{array}$ & $8.68 \pm 6.50$ & $7.34 \pm 4.41$ & 1.3 & $0.570^{9}$ \\
\hline $\begin{array}{l}\text { Number of hospitalisations ( } \mathrm{n} \text {; mean } \\
\pm \mathrm{s} \text { )] }\end{array}$ & $79 ; 0.77 \pm 1.39$ & $72 ; 0.73 \pm 1.23$ & & \\
\hline Rate (per 100 person-years) & 77.5 & 72.7 & $1.07(0.66 ; 1.72)$ & $0.796^{\#}$ \\
\hline Days rate (per 100 person-years) & 6.7 & 5.2 & $1.26(0.73 ; 2.17)$ & $0.405^{\#}$ \\
\hline Patients with $\geqslant 1$ hospitalisation & $\mathrm{n}=37$ & $\mathrm{n}=41$ & $0.88(0.62 ; 1.24)$ & $0.455^{+}$ \\
\hline $\begin{array}{l}\text { Duration per hospitalisation days } \\
\text { (mean } \pm \text { SD) }\end{array}$ & $9.36 \pm 7.63$ & $6.99 \pm 4.34$ & 2.4 & $0.235^{\Uparrow}$ \\
\hline
\end{tabular}


TABLE 7 Secondary outcome mean changes from baseline at 6 and 12 months of follow-up in the self-management and usual care group

\begin{tabular}{|c|c|c|c|c|c|}
\hline & \multicolumn{2}{|c|}{ Self-management } & \multicolumn{2}{|c|}{ Usual care } & $\begin{array}{c}\text { Self-management } \\
\text { versus usual care } \\
\Delta \text { between-group } \\
\text { difference }(95 \% \mathrm{CI})\end{array}$ \\
\hline Dyspnoea score (mMRC) ${ }^{\#}$ & 0.07 (83) & $0.28(80)$ & $0.03(76)$ & $0.08(78)$ & $0.21(-0.09 ; 0.50)$ \\
\hline FEV $_{1} \%$ predicted ी & N/A & $-1.71(81)$ & N/A & $-0.61(75)$ & $-1.09(-3.52 ; 1.33)$ \\
\hline $\mathrm{FEV}_{1} / \mathrm{FVC}^{\text {ๆ }}$ & $\mathrm{N} / \mathrm{A}$ & $-0.06(81)$ & $\mathrm{N} / \mathrm{A}$ & $-0.80(75)$ & $0.69(-1.43 ; 2.81)$ \\
\hline CRQ dyspnoea? & $-0.23(83)$ & $-0.29(79)$ & $-0.14(77)$ & $-0.03(80)$ & $-0.26(-0.59 ; 0.08)$ \\
\hline CRQ fatigue ? $^{-1}$ & $-0.23(83)$ & $-0.09(79)$ & $0.20(77)$ & $0.12(80)$ & $-0.20(-0.49 ; 0.10)$ \\
\hline CRQ emotional function " & $-0.15(83)$ & $-0.10(79)$ & $0.19(77)$ & $0.30(80)$ & $-0.41(-0.70 ;-0.11)^{+}$ \\
\hline CRQ mastery & $-0.07(83)$ & $0.11(79)$ & $0.22(77)$ & $0.30(80)$ & $-0.18(-0.48 ; 0.11)$ \\
\hline ICFS fatigue experiences $\#$ & $2.02(83)$ & $1.63(77)$ & $-3.85(76)$ & $-1.62(79)$ & $3.11(-0.79 ; 7.03)$ \\
\hline ICFS fatigue impacts ${ }^{\#}$ & $5.03(84)$ & $3.04(77)$ & $-3.25(76)$ & $-0.81(80)$ & $3.04(-2.56 ; 8.64)$ \\
\hline CSES negative affects $\#$ & $-0.04(66)$ & $-0.10(66)$ & $0.04(68)$ & $0.03(70)$ & $-0.16(-0.37 ; 0.06)$ \\
\hline PIH total & N/A & $2.90(78)$ & N/A & $2.89(79)$ & $0.28(-2.43 ; 3.00)$ \\
\hline PIH knowledge and coping? & $\mathrm{N} / \mathrm{A}$ & $2.17(78)$ & N/A & $1.99(79)$ & $0.47(-1.30 ; 2.23)$ \\
\hline $\begin{array}{l}\text { PIH symptom management, } \\
\text { adherence to treatment }\end{array}$ & $\mathrm{N} / \mathrm{A}$ & $0.73(78)$ & $\mathrm{N} / \mathrm{A}$ & $0.90(79)$ & $0.18(-1.33 ; 1.68)$ \\
\hline HADS anxiety\# & $-0.41(82)$ & $-0.76(79)$ & $-0.91(76)$ & $-1.15(80)$ & $0.22(-0.77 ; 1.21)$ \\
\hline HADS depression ${ }^{\#}$ & $-0.13(82)$ & $-0.34(79)$ & $-0.59(79)$ & $-0.49(80)$ & $0.15(-0.68 ; 0.97)$ \\
\hline
\end{tabular}

CAT: COPD Assessment Test; CRQ: Chronic Respiratory Disease Questionnaire; CSES: COPD Self-Efficacy Scale; FEV ${ }_{1}$ : forced expiratory volume in $1 \mathrm{~s}$; FVC: forced (expiratoryl vital capacity; HADS: Hospital Anxiety Depression Scale; ICFS: Identity-Consequence Fatigue Score; mMRC: modified Medical Research Council dyspnoea scale; PIH: Partners in Health scale; VAS: Visual Analogue Scale. N/A: not applicable. \#: higher score indicates worse outcome; ${ }^{\uparrow}$ : higher score indicates better outcome; ${ }^{+}$: statistically significant at a $p<0.05$ level; linear mixed-effect models for repeated measurements analyses.

hospitalisations and mortality were detected. Our results demonstrate that COPD patients may benefit from using exacerbation action plans that are tailored for comorbidities.

In this study, the number of COPD exacerbation days per patient per year (primary outcome) was numerically lower in the self-management group (9.6 days) compared to UC (15.6 days); however, this difference was not statistically significant. The severity per exacerbation day was also numerically lower, although not statistically significant, in the self-management group (median 4.1) compared to UC (median 5.0). This may be because the study was underpowered. The mean number of 34 exacerbation days per patient per year in the UC control group was much lower than the 64 a priori anticipated. Our power calculation was based on the COPE-II study results of patients without severe comorbidities [9]. Reasonably, one would have expected an even higher rate of exacerbations in the COPE-III population with comorbidities. Improvements in UC over time may be an explanation for the improved exacerbation rate [10]. Whereas all patients had at least three exacerbations or one respiratory-related hospitalisation in the 2 years preceding study entry, one-third of the patients in the self-management group had no COPD exacerbation during the 12-month follow-up period, and could therefore not have benefitted from the action plan. The effect of improvement of UC and consequences for trial designs (i.e. less room for improvement) should be carefully considered in designing future studies of COPD interventions. This is also underlined by another recently published COPD self-management study that reported no effect in the primary outcome due to much lower event rates in the control group than anticipated [24].

There was no significant between-group difference in the number of COPD exacerbations. This was expected as action plans were only activated after the onset of an exacerbation. Self-management was associated with quicker initiation of prednisolone courses, which may have contributed to an earlier initiation of appropriate self-treatment of deteriorating COPD symptoms and therefore a shorter duration of COPD exacerbations. However, it is not possible to definitively determine which component(s) of the exacerbation action plan were responsible for the positive effects on exacerbation days and duration. 
Whereas this study was not powered to detect an effect of exacerbation action plans on hospitalisations, we observed a significant reduced risk of having at least one respiratory-related hospitalisation during follow-up in self-management patients. This effect was larger than in our previous study in COPD patients without comorbidities [9]. Non-respiratory and cardiac-related hospitalisations, and exacerbations in comorbid conditions, were observed more often in the self-management group, although these differences were not statistically significant. Since we did not power for exacerbations in comorbid conditions, conclusions from these results are limited. The numerical increase in non-respiratory and cardiac-related hospitalisations may, however, be explained by the relevant action plans. These directed self-management group patients to contact a case manager or healthcare provider for support if the cause of symptom deterioration was unclear. This was for safety reasons; its importance was highlighted by a study that was prematurely terminated due to excess all-cause mortality in the intervention group [25]. While our study was not powered to assess mortality, all-cause, cardiac- and respiratory-related deaths were all lower in the self-management group, providing some evidence that multimodal action plans with ongoing case-manager support are safe. Forthcoming cost analyses will assess the cost-effectiveness of the self-management intervention as the majority of costs in COPD patients with comorbidities are related to treatment of exacerbations, with hospitalisation costs a major component $[4,26]$.

The self-management intervention improved patients' self-efficacy in preventing breathing difficulty, but decreased patients' perceptions of emotional health, albeit without negative changes in overall emotional function (HADS scores). Behaviour change techniques (i.e. goal setting, action planning, self-monitoring) [27] were used to improve patient self-regulation skills as well as targeted uptake and optimal use of appropriate self-management behaviours. The lower emotional function scores may have resulted from patients' greater awareness of emotional symptoms associated with their health status, and this may be reflected in their responses in the CRQ domain. However, one should be cautious with the interpretation of these findings as they are not supported by other secondary outcomes.

Tailored approaches with individualised care plans are required to reduce treatment burden and optimise care for patients with COPD and comorbidities [28]. Efforts to improve this have focused on standardised care for each disease [29]. However, for COPD patients with multiple morbidities, these disease-specific recommendations may be inappropriate and lead to an excessive patient burden (e.g. increased exacerbations and length of hospitalisations) [29]. Although previous studies on COPD self-management interventions were not all without comorbidities [10], those studies used COPD-specific action plans and did not account for treatment adjustments related to comorbidities. The results of the current study should encourage healthcare providers to monitor comorbidities in COPD self-management interventions and exacerbation action plans, targeting timely treatment actions for each disease, and anticipating better treatment effectiveness and increased safety.

Our RCT has several strengths. This is the first study in which COPD exacerbation action plans that included actions for comorbidities have been evaluated. By including COPD patients with comorbidities our patient group is typical of those treated in usual practice. Ongoing case manager support, highlighted as a key component in reducing COPD (re)admissions [30] and emphasised as critical in the latest COPD self-management review regarding exacerbation plans [12], was offered to reinforce the patients' decision-making and targeted behavioural change. While our self-management intervention included case manager phone support, it was not a case-management programme, but a self-management intervention since the patient was in control through patient-initiated actions [6]. Two recent published studies showed reduced mortality in more complex COPD patients (those having comorbidities, frequent hospitalisations and/or exacerbations) who were offered intensive case-management interventions [24, 31]. Another strength of our study was the use of symptom diaries with a high completion rate (59629 diary days; $81.3 \%$ ), comparable to another multicentre RCT of exacerbation action plans [11]. Moreover, the diaries enabled calculation of daily symptom scores, minimised recall bias and yielded information on day-to-day variability [32].

Our study also has limitations. First, the baseline 6MWD in the self-management group was $48 \mathrm{~m}$ shorter, which is clinically significant and has been related to poorer outcomes, including increased mortality [33]. This baseline between-group difference in 6MWD is, however, likely to have resulted in under- and not overestimation of the benefits of self-management. Second, contact with case managers per se could have produced positive effects. However, while $52.9 \%$ of the self-management patients reported contact with the study office, the majority of the contacts were regarding logistics (e.g. check appointment dates, ask for a new diary). Third, a small number of patients with a long exacerbation duration may not have properly adjusted their individual symptom levels on their "What are my 'usual' symptoms" card, and/or may not have fully recovered from their exacerbation [34]. A significant change in COPD symptoms (meeting our pre-defined definition of an acute COPD exacerbation) may have converted into a stepwise chronic deterioration of symptoms that was not recognised as such by the case managers. This highlights a 
potential limitation of the use of paper versions of symptom diaries. Real-time symptom monitoring could be a more effective way to help patients and healthcare providers to recognise when recovery after an exacerbation is prolonged. Furthermore, algorithms could be used to indicate that individual baseline "usual" symptoms need to be redefined. However, there were only two patients with extremely long COPD exacerbation durations ( $>300$ days) and sensitivity analyses excluding these patients did not change results. Fourth, because of the nature of the self-management intervention, blinding of patients to group assignment was not possible, potentially introducing performance bias (e.g. more symptom awareness and recognition in the self-management group compared to UC). Fifth, many patients declined participation (42\%), often because of the logistic issues and time commitments associated with a RCT. Furthermore, the need for patients to be in a stable phase at the start of the study hampered patient inclusion because COPD exacerbations or flare-ups of comorbidities are common in this complex patient population. However, if this self-management intervention was included as part of usual clinical practice, fewer barriers would be in place to preclude its use. Finally, we cannot define which parts of the intervention may have been driven by the study effects.

In summary, although the number of COPD exacerbation days was not significantly lower in the self-management group, this study provides new evidence that exacerbation action plans for COPD patients with comorbidities embedded in an individualised, multi-faceted self-management intervention could be an effective tool to reduce the duration of COPD exacerbations and the risk of having a respiratory-related hospitalisation, without excess all-cause mortality. For the implementation of a successful self-management intervention for patients with COPD and comorbidities we recommend using a patient-tailored approach that includes an individual assessment of COPD and comorbidities, information on self-management, daily self-monitoring of symptoms, exacerbation action plans for COPD and comorbidities, and case manager support for feedback and motivation.

Acknowledgements: We would like to thank all the COPD patients who participated in this study. We would also like to thank all disease experts in respiratory and cardiac disease, diabetes, and mental health for their expertise regarding the development and adjustments of the action plans. Furthermore, we would like to thank the data managers and the nurses for their support in running this study. Finally, we thank the members of the Data and Safety Monitoring Board.

Support statement: This study was supported by the Lung Foundation Netherlands (grant number 3.4.11.061), Lung Foundation Australia (Australian Lung Foundation Boehringer Ingelheim COPD Research Fellowship 2010), Repat Foundation, GlaxoSmithKline (unrestricted grant) and Stichting Astma Bestrijding. Funding information for this article has been deposited with the Crossref Funder Registry.

Conflict of interest: A. Lenferink reports grants from Stichting Astmabestrijding (SAB) and GlaxoSmithKline (unrestricted grant), during the conduct of the study. J. van der Palen reports grants from Netherlands Lung Foundation, during the conduct of the study. P.D.L.P.M. van der Valk has nothing to disclose. P. Cafarella has nothing to disclose. A. van Veen has nothing to disclose. S. Quinn has nothing to disclose. C.G.M. Groothuis-Oudshoorn has nothing to disclose. M.G. Burt has nothing to disclose. M. Young has nothing to disclose. P.A. Frith has nothing to disclose. T.W. Effing reports grants from The Repat Foundation, Australian Lung Foundation and Dutch Asthma Foundation, during the conduct of the study.

\section{References}

1 Anecchino C, Rossi E, Fanizza C, et al. Prevalence of chronic obstructive pulmonary disease and pattern of comorbidities in a general population. Int J Chron Obstruct Pulmon Dis 2007; 2: 567-574.

2 Vanfleteren LE, Spruit MA, Groenen M, et al. Clusters of comorbidities based on validated objective measurements and systemic inflammation in patients with chronic obstructive pulmonary disease. Am J Respir Crit Care Med 2013; 187: 728-735.

3 Huber MB, Wacker ME, Vogelmeier CF, et al. Comorbid influences on generic health-related quality of life in COPD: a systematic review. PLoS One 2015; 10: e0132670.

4 Chen W, FitzGerald JM, Sin DD, et al. Excess economic burden of comorbidities in COPD: a 15-year population-based study. Eur Respir J 2017; 50: 1-10.

5 Hillas G, Perlikos F, Tsiligianni I, et al. Managing comorbidities in COPD. Int J Chron Obstruct Pulmon Dis 2015; 10: $95-109$.

6 Effing TW, Vercoulen JH, Bourbeau J, et al. Definition of a COPD self-management intervention: International Expert Group consensus. Eur Respir J 2016; 48: 46-54.

7 Zwerink M, Brusse-Keizer M, van der Valk PD, et al. Self management for patients with chronic obstructive pulmonary disease. Cochrane Database Syst Rev 2014; 19: CD002990.

8 Bourbeau J, van der Palen J. Promoting effective self-management programmes to improve COPD. Eur Respir J 2009; 33: 461-463.

9 Effing T, Kerstjens $\mathrm{H}$, van der Valk $\mathrm{P}$, et al. (Cost)-effectiveness of self-treatment of exacerbations on the severity of exacerbations in patients with COPD: the COPE II study. Thorax 2009; 64: 956-962.

10 Lenferink A, Brusse-Keizer M, van der Valk PD, et al. Self-management interventions including action plans for exacerbations versus usual care in patients with chronic obstructive pulmonary disease. Cochrane Database Syst Rev 2017; 8: CD011682.

11 Trappenburg JC, Monninkhof EM, Bourbeau J, et al. Effect of an action plan with ongoing support by a case manager on exacerbation-related outcome in patients with COPD: a multicentre randomised controlled trial. Thorax 2011; 66: 977-984. 
12 Howcroft M, Walters EH, Wood-Baker R, et al. Action plans with brief patient education for exacerbations in chronic obstructive pulmonary disease. Cochrane Database Syst Rev 2016; 12: CD005074.

13 Morgan MD. Action plans for COPD self-management. Integrated care is more than the sum of its parts. Thorax 2011; 66: 935-936.

14 Lenferink A, Frith $\mathrm{P}$, van der Valk $\mathrm{P}$, et al. A self-management approach using self-initiated action plans for symptoms with ongoing nurse support in patients with Chronic Obstructive Pulmonary Disease (COPD) and comorbidities: the COPE-III study protocol. Contemp Clin Trials 2013; 36: 81-89.

15 Effing TW, Lenferink A, Buckman J, et al. Development of a self-treatment approach for patients with COPD and comorbidities: an ongoing learning process. J Thorac Dis 2014; 6: 1597-1605.

16 Dickstein K, Cohen-Solal A, Filippatos G, et al. ESC guidelines for the diagnosis and treatment of acute and chronic heart failure 2008: the Task Force for the diagnosis and treatment of acute and chronic heart failure 2008 of the European Society of Cardiology. Developed in collaboration with the Heart Failure Association of the ESC (HFA) and endorsed by the European Society of Intensive Care Medicine (ESICM). Eur Heart J 2008; 29: $2388-2442$

17 Zigmond AS, Snaith RP. The Hospital Anxiety and Depression Scale. Acta Psychiatr Scand 1983; 67: 361-370.

18 Spinhoven P, Ormel J, Sloekers PP, et al. A validation study of the Hospital Anxiety and Depression Scale (HADS) in different groups of Dutch subjects. Psychol Med 1997; 27: 363-370.

19 Altman DG, Bland JM. Treatment allocation by minimisation. BMJ 2005; 330: 843.

20 Anthonisen NR, Manfreda J, Warren CP, et al. Antibiotic therapy in exacerbations of chronic obstructive pulmonary disease. Ann Intern Med 1987; 106: 196-204.

21 Rodriguez-Roisin R. Toward a consensus definition for COPD exacerbations. Chest 2000; 117: 5 Suppl. 2, 398S-401S.

22 Begg $\mathrm{C}$, Cho M, Eastwood S, et al. Improving the quality of reporting of randomized controlled trials. The CONSORT statement. JAMA 1996; 276: 637-639.

23 Newgard CD, Lewis RJ. Missing data: how to best account for what is not known. JAMA 2015; 314: 940-941.

24 Kessler R, Casan-Clara P, Koehler D, et al. COMET: a multicomponent home-based disease-management programme versus routine care in severe COPD. Eur Respir J 2018; 51: 1-14.

25 Fan VS, Gaziano JM, Lew R, et al. A comprehensive care management program to prevent chronic obstructive pulmonary disease hospitalisations: a randomized, controlled trial. Ann Intern Med 2012; 156: 673-683.

26 van Boven JFM. Costly comorbidities of COPD: the ignored side of the coin? Eur Respir J 2017; 50: 1700917.

27 Michie S, Richardson M, Johnston M, et al. The behavior change technique taxonomy (v1) of 93 hierarchically clustered techniques: building an international consensus for the reporting of behavior change interventions. Ann Behav Med 2013; 46: 81-95.

28 Vanfleteren LE, Spruit MA, Franssen FM. Tailoring the approach to multimorbidity in adults with respiratory disease: the NICE guideline. Eur Respir J 2017; 49: 1601696.

29 Salisbury C, Man MS, Bower P, et al. Management of multimorbidity using a patient-centred care model: a pragmatic cluster-randomised trial of the 3D approach. Lancet 2018; 392: 41-50.

30 Benzo R, Vickers K, Novotny PJ, et al. Health coaching and COPD rehospitalization: a randomized study. Am J Respir Crit Care Med 2016; 194: 672-680.

31 Rose L, Istanboulian L, Carriere L, et al. Program of Integrated Care for Patients with Chronic Obstructive Pulmonary Disease and Multiple Comorbidities (PIC COPD+): a randomised controlled trial. Eur Respir J 2018; 51: 1701567.

32 Leidy NK, Sexton CC, Jones PW, et al. Measuring respiratory symptoms in clinical trials of COPD: reliability and validity of a daily diary. Thorax 2014; 69: 443-449.

33 Spruit MA, Polkey MI, Celli B, et al. Predicting outcomes from 6-minute walk distance in chronic obstructive pulmonary disease. J Am Med Dir Assoc 2012; 13: 291-297.

34 Wageck B, Cox NS, Holland AE. Recovery following acute exacerbations of chronic obstructive pulmonary disease a review. COPD 2019; 16: 93-103. 